\title{
Quantitative Voronovskaya-Type Theorems for Fejér-Korovkin Operators
}

\author{
Jorge BustamantE* AND LÁZARO Flores-DE-JEsús
}

\begin{abstract}
In recent times, quantitative Voronovskaya type theorems have been presented in spaces of non-periodic continuous functions. In this work, we proved similar results but for Fejér-Korovkin trigonometric operators. That is we measure the rate of convergence in the associated Voronovskaya type theorem. Recall that these operators provide the optimal rate in approximation by positive linear operators. For the proofs, we present new inequalities related with trigonometric polynomials as well as with the convergence factor of the Fejér-Korovkin operators. Our approach includes spaces of Lebesgue integrable functions.
\end{abstract}

Keywords: Fejér-Korovkin operators, quantitative Voronovskaya type theorems.

2020 Mathematics Subject Classification: 41A36, 41A25, 42A10.

\section{INTRODUCTION}

In recent times, there have been some interests in studying quantitative Voronovskaya-type theorems, but almost all the papers are concerned with positive linear operators in spaces of non-periodic functions. The methods used in those papers are not useful in dealing with periodic functions for two reasons (at least). First they use different kinds of Taylor's formula and second, in the non-periodical case do not appear conjugate functions.

It is known that the Voronovskaya-type theorems are related with the saturation class of some families of operators. We have noticed that in the case of trigonometric polynomial approximation process the Voronovskaya-type theorems depend on particular properties of the operators. In [1], the authors considered this kind of problem for Fejér sums. In this paper, we consider the Fejér-Korovkin operators.

Let $C_{2 \pi}$ denote the Banach space of all $2 \pi$-periodic, continuous functions $f$ defined on the real line $\mathbb{R}$ with the sup norm

$$
\|f\|_{\infty}=\max _{x \in[-\pi, \pi]}|f(x)| .
$$

For $1 \leq p<\infty$, the Banach space $\mathbb{L}^{p}$ consists of all $2 \pi$-periodic, $p$-th power Lebesgue integrable functions $f$ on $\mathbb{R}$ with the norm

$$
\|f\|_{p}=\left(\frac{1}{2 \pi} \int_{-\pi}^{\pi}|f(x)|^{p} d x\right)^{1 / p} .
$$

In order to simplify, we write $X^{p}=\mathbb{L}^{p}$ for $1 \leq p<\infty$ and $X^{\infty}=C_{2 \pi}$. By $W_{p}^{r}$, we mean the family of all functions $f \in X^{p}$ such that $f, \ldots, D^{r-1}(f)$ are absolutely continuous and 
$D^{r}(f) \in X^{p}$. Here, $D(f)=D^{1}(f)=f^{\prime}$ and $D^{r+1}(f)=D\left(D^{r}(f)\right)$. Recall that for $f \in \mathbb{L}^{1}$ and $k \in \mathbb{N}_{0}$, the Fourier coefficients are defined by

$$
a_{k}(f)=\frac{1}{\pi} \int_{-\pi}^{\pi} f(t) \cos (k t) d t \quad \text { and } \quad b_{k}(f)=\frac{1}{\pi} \int_{-\pi}^{\pi} f(t) \sin (k t) d t,
$$

and the (formal) Fourier series is given by

$$
f(x) \sim \frac{a_{0}(f)}{2}+\sum_{k=1}^{\infty}\left(a_{k}(f) \cos (k x)+b_{k}(f) \sin (k x)\right):=\sum_{k=0}^{\infty} A_{k}(f) .
$$

For $n \in \mathbb{N}$, the Fejér-Korovkin kernel is defined by

$$
K_{n}(x)=\frac{1}{n+2} \sin ^{2} \frac{\pi}{n+2} \frac{\cos ^{2}((n+2) x / 2)}{(\cos (\pi /(n+2))-\cos x)^{2}}
$$

for $x \neq \pm \pi /(n+2)+2 j \pi, j \in \mathbb{Z}$. For $f \in X^{1}$ and $n \in \mathbb{N}$, the Fejér-Korovkin operator is defined by

$$
\mathbb{F}_{n}(f, x)=\frac{1}{2 \pi} \int_{-\pi}^{\pi} f(x+t) K_{n}(t) d t .
$$

Some Voronovskaya type theorems for the operators $\mathbb{F}_{n}$ are known.

Theorem 1.1. (Korovkin, [6]) If $f \in C_{2 \pi}, x \in[-\pi, \pi]$ and $f^{\prime \prime}(x)$ exists, then

$$
\mathbb{F}_{n}(f, x)-f(x)=\frac{\pi^{2}}{2 n^{2}} f^{\prime \prime}(x)+o\left(n^{-2}\right) .
$$

Theorem 1.2. (Butzer and Görlich, [2, page 385]) If $1<p<\infty$ and $f \in W_{p}^{2}$, then

$$
\lim _{n \rightarrow \infty}\left\|n^{2}\left(\mathbb{F}_{n}(f)-f\right)-\frac{\pi^{2}}{2} f^{\prime \prime}\right\|_{p}=0 .
$$

The main purpose of the paper is to present a quantitative Voronovskaya-type theorem for the operators $\mathbb{F}_{n}$. That is we want to estimate the rate of convergence to zero in the results presented above. This will be accomplished in the last section of the article, where the case $p=1$ is also included.

The work is organized as follows. In Section 2, we include a collection of known definitions and results which will be used later. For instance, in the non-periodic case conjugate functions are not needed, but for our approach they are important. In Section 3, we prove some inequalities related with trigonometric polynomials (we think that they have independent interest). Section 4 is very technical. It involves complicated computations related with the convergence factors of Fejér-Korovkin operators. In Section 5, we include the main result (Theorem 5.7). The most important idea is to prove first a Voronovskaya theorem limited to polynomials (Proposition 5.8).

\section{KNOWN RESULTS}

The convolution of $f, g \in \mathbb{L}^{1}, g$ an even function, is defined by

$$
(f * g)(x)=\frac{1}{\pi} \int_{-\pi}^{\pi} f(x+t) g(t) d t=\frac{1}{\pi} \int_{0}^{\pi}(f(x+t)+f(x-t)) g(t) d t .
$$

It is known that if $f \in X^{p}$ and $g \in X^{1}$, then $f * g \in X^{p}$ and

$$
\|f * g\|_{p} \leq\|g\|_{1}\|f\|_{p} .
$$


For $f \in X^{1}$, the conjugate function is defined by

$$
\widetilde{f}(x)=-\frac{1}{2 \pi} \int_{0}^{\pi} \frac{f(x+t)-f(x-t)}{\tan (t / 2)} d t=-\lim _{\varepsilon \rightarrow 0} \frac{1}{2 \pi} \int_{\varepsilon}^{\pi} \frac{f(x+t)-f(x-t)}{\tan (t / 2)} d t,
$$

whenever the limit exists. It is known that if $f \in X^{p}$ with $1<p<\infty$, then $\widetilde{f} \in X^{p}$, and that is not the case for $p=1$ and $p=\infty$. Recall that for $n \in \mathbb{N}$ and $f \in X^{1}$, the Fejér sum of order $n$ is defined by

$$
\sigma_{n}(f, x)=\sum_{k=0}^{n}\left(1-\frac{k}{n+1}\right) A_{k}(f, x) .
$$

Throughout the paper, we use the following notations. $\mathbb{T}_{n}$ denotes the family of all trigonometric polynomials of degree no greater that $n$, and $\mathbb{T}_{n}^{0}$ is the family of all $T \in \mathbb{T}_{n}$ with mean zero, that is

$$
\int_{-\pi}^{\pi} T_{n}(x) d x=0
$$

Proposition 2.1. ([1, Prop. 2.4]) For each $n, r \in \mathbb{N}$ and $T \in \mathbb{T}_{n}$, one has

$$
\left(I-\sigma_{n}\right)^{r}(T)=\left\{\begin{array}{cc}
\frac{(-1)^{r / 2}}{(n+1)^{r}} D^{r}(T), & \text { r even }, \\
\frac{(-1)^{(r-1) / 2}}{(n+1)^{r}} D^{r}(\widetilde{T}), & r \text { odd } .
\end{array}\right.
$$

Theorem 2.3. ([9, p. 215]) If $1 \leq p \leq \infty, r, n \in \mathbb{N}$ and $T \in \mathbb{T}_{n}$, then

$$
\left\|D^{r}(T)\right\|_{p} \leq\left(\frac{n}{2 \sin (n h / 2)}\right)^{r}\left\|\Delta_{h}^{r} T\right\|_{p}
$$

for any $h \in(0,2 \pi / n)$. Moreover $\left\|D^{r}(\widetilde{T})\right\|_{p} \leq n^{r}\|T\|_{p}$.

For $r \in \mathbb{N}$, a function $f \in X^{p}$, and $h>0$, the usual modulus of smoothness of order $r$ is defined by

$$
\omega_{r}(f, t)_{p}=\sup _{|h| \leq t}\left\|\left(I-T_{h}\right)^{r}(f)\right\|_{p},
$$

where $T_{h}(f, x)=f(x+h)$ is the translation operator. We also use the notations $\Delta_{h}^{r} f(x)=$ $\left(I-T_{h}\right)^{r}(f)$. For $1 \leq p \leq \infty$ and $f \in X^{p}$, the best approximation of $f$ by elements of $\mathbb{T}_{n}$ is defined by

$$
E_{n, p}(f)=\inf _{T \in \mathbb{T}_{n}}\|f-T\|_{p}
$$

Theorem 2.4. (Foucart et al, [5, Theorem 2.5]) If $1 \leq p \leq \infty, f \in X^{p}$, and $n \in \mathbb{N}$, then

$$
E_{n, p}(f) \leq 5 \omega_{1}\left(f, \frac{2 \pi}{n+1}\right)_{p} .
$$

The following result is easy to prove (see [3, p. 77]).

Proposition 2.2. Assume $1 \leq p \leq \infty$ and

$$
Q_{n}(x)=\frac{1}{2}+\sum_{k=1}^{n} \lambda_{k, n} \cos (k x)
$$

is a non-negative trigonometric polynomial. If $g \in W_{p}^{2}$, then

$$
\left\|g-g * Q_{n}\right\|_{p} \leq \frac{\pi^{2}}{2}\left(1-\lambda_{1, n}\right)\left\|D^{2}(g)\right\|_{p} .
$$


We need some results taken from the Zygmund book [10, pages 93 and 183].

Proposition 2.3. If $\left\{c_{n}\right\}$ is a convex and bounded sequence, then $\left\{c_{n}\right\}$ decreases, $n \Delta^{1} c_{n} \rightarrow 0$, and

$$
\sum_{n=0}^{\infty}(n+1) \Delta^{2} c_{n}=c_{0}-\lim _{n \rightarrow \infty} c_{n} .
$$

Proposition 2.4. If $\left\{c_{n}\right\}$ is a convex sequence which converges to zero, then the series

$$
\frac{c_{0}}{2}+\sum_{k=1}^{\infty} c_{k} \cos (k x)
$$

converges for all $x \neq 0$ to a nonnegative and integrable function.

\section{INEQUALITIES FOR TRIGONOMETRIC POLYNOMIALS}

Proposition 3.5. The function

$$
\varphi(x)=\sum_{k=1}^{\infty} \frac{\cos (k x)}{k}, \quad x \neq 0,
$$

is integrable. Moreover

$$
\|\varphi\|_{1} \leq \frac{3}{2}
$$

Proof. If we consider Proposition 2.4 with the sequence $\left\{a_{n}\right\}$, given by $a_{0}=3 / 2$ and $a_{k}=1 / k$ for $k \in \mathbb{N}$, then

$$
\frac{3}{4}+\sum_{k=1}^{\infty} \frac{\cos (k x)}{k} \geq 0
$$

for $x \neq 0$. But

$$
\begin{aligned}
\frac{1}{2 \pi} \int_{-\pi}^{\pi}\left|\sum_{k=1}^{\infty} \frac{\cos (k x)}{k}\right| d x & \leq \frac{3}{4}+\frac{1}{2 \pi} \int_{-\pi}^{\pi}\left|\frac{3}{4}+\sum_{k=1}^{\infty} \frac{\cos (k x)}{k}\right| d x \\
& =\frac{3}{4}+\frac{1}{2 \pi} \int_{-\pi}^{\pi}\left(\frac{3}{4}+\sum_{k=1}^{\infty} \frac{\cos (k x)}{k}\right) d x \\
& =\frac{3}{4}+\frac{1}{2} \sum_{k=0}^{\infty}(k+1) \Delta^{2} a_{k} \\
& =\frac{3}{2},
\end{aligned}
$$

where we use Proposition 2.3.

Remark 3.1. In the proof of the previous Proposition, we can not take $a_{0}<3 / 2$, because we need that $\Delta^{2} a_{0}=a_{0}-2 a_{1}+a_{2}=a_{0}-3 / 2 \geq 0$.

Theorem 3.5. For $1 \leq p \leq \infty$, each $n \in \mathbb{N}$ and $T \in \mathbb{T}_{n}$, one has

$$
\left\|\left(I-\sigma_{n}\right)(T)\right\|_{p} \leq \frac{3(n+1)}{2}\left\|\left(I-\sigma_{n}\right)^{2}(T)\right\|_{p},
$$

where $\sigma_{n}$ denotes the Fejér sum. Moreover, if $T \in \mathbb{T}_{n}^{0}$, then

$$
\|T\|_{p} \leq \frac{3(n+1)}{2}\left\|\left(I-\sigma_{n}\right)(T)\right\|_{p}
$$


Proof. Define $\tau_{n}: \mathbb{T}_{n}^{0} \rightarrow \mathbb{T}_{n}^{0}$ by the equation

$$
\tau_{n}(T)=(n+1) \sum_{k=1}^{n} \frac{A_{k}(x)}{k},
$$

where $T(x)=\sum_{k=1}^{n} A_{k}(x)$. Notice that, for each $T \in \mathbb{T}_{n}^{0}$, it follows from (2.1) that

$$
\left\|\tau_{n}(T)\right\|_{p} \leq(n+1)\|\varphi\|_{1}\|T\|_{p} \leq \frac{3(n+1)}{2}\|T\|_{p},
$$

where $\varphi$ is the function in Proposition 3.5. On the other hand, if $T$ is given as in (3.4), then

$$
\tau_{n}\left(\left(I-\sigma_{n}\right)(T), x\right)=\tau_{n}\left(\sum_{k=1}^{n} \frac{k}{n+1} A_{k}, x\right)=\sum_{k=1}^{n} A_{k}(x)=T(x) .
$$

Therefore, for any $T \in \mathbb{T}_{n}$, if we set $T^{*}=T-A_{0}(T) / 2$, then

$$
\begin{aligned}
\left\|\left(I-\sigma_{n}\right)(T)\right\|_{p} & =\left\|\left(I-\sigma_{n}\right)\left(T^{*}\right)\right\|_{p} \\
& =\| \tau_{n}\left(\left(I-\sigma_{n}\right)^{2}\left(T^{*}\right) \|_{p}\right. \\
& \leq \frac{3(n+1)}{2}\left\|\left(I-\sigma_{n}\right)^{2}\left(T^{*}\right)\right\|_{p} \\
& =\frac{3(n+1)}{2}\left\|\left(I-\sigma_{n}\right)^{2}(T)\right\|_{p} .
\end{aligned}
$$

Finally, if $T \in \mathbb{T}_{n}^{0}$, it follows from (3.5) that

$$
\|T\|_{p}=\left\|\tau_{n}\left(\left(I-\sigma_{n}\right)(T)\right)\right\|_{p} \leq \frac{3(n+1)}{2}\left\|\left(I-\sigma_{n}\right)(T)\right\|_{p} .
$$

Intermediate derivatives have been used by several authors. Here, we present some particular constants.

Corollary 3.1. If $n \in \mathbb{N}$ and $T \in \mathbb{T}_{n}^{0}$, then

and

$$
\begin{gathered}
\|T\|_{p} \leq \frac{9}{4}\left\|T^{\prime \prime}\right\|_{p}, \quad\|T\|_{p} \leq \frac{27}{8}\left\|D^{3}(\widetilde{T})\right\|_{p}, \\
\left\|\widetilde{T}^{\prime}\right\|_{p} \leq \frac{3}{2}\left\|T^{\prime \prime}\right\|_{p}, \quad\left\|\widetilde{T}^{\prime}\right\|_{p} \leq \frac{9}{4}\left\|D^{3}(\widetilde{T})\right\|_{p}
\end{gathered}
$$

$$
\left\|D^{3}(\widetilde{T})\right\|_{p} \leq 2(n+1)\left\|T^{\prime \prime}\right\|_{p} .
$$

Proof. The result follows from Theorem 3.5 and Proposition 2.1. For instance,

$$
\|T\|_{p} \leq \frac{3(n+1)}{2}\left\|\left(I-\sigma_{n}\right)(T)\right\|_{p} \leq \frac{9(n+1)^{2}}{4}\left\|\left(I-\sigma_{n}\right)^{2}(T)\right\|_{p}=\frac{9}{4}\left\|T^{\prime \prime}\right\|_{p}
$$

and

On the other hand,

$$
\|T\|_{p} \leq \frac{27(n+1)^{3}}{8}\left\|\left(I-\sigma_{n}\right)^{3}(T)\right\|_{p}=\frac{27}{8}\left\|D^{3}(\widetilde{T})\right\|_{p} .
$$

$$
\left\|\widetilde{T}^{\prime}\right\|_{p}=(n+1)\left\|\left(I-\sigma_{n}\right)(T)\right\|_{p} \leq \frac{3}{2}(n+1)^{2}\left\|\left(I-\sigma_{n}\right)^{2}(T)\right\|_{p}=\frac{3}{2}\left\|T^{\prime \prime}\right\|_{p}
$$

and

$$
\left\|\widetilde{T}^{\prime}\right\|_{p} \leq \frac{9}{4}(n+1)^{3}\left\|\left(I-\sigma_{n}\right)^{3}(T)\right\|_{p}=\frac{9}{4}\left\|D^{3}(\widetilde{T})\right\|_{p} .
$$


Finally,

$$
\left\|\widetilde{T}^{\prime \prime \prime}\right\|_{p}=\left\|\left(\widetilde{T^{\prime \prime}}\right)^{\prime}\right\|_{p}=(n+1)\left\|\left(I-\sigma_{n}\right)\left(T^{\prime \prime}\right)\right\|_{p} \leq 2(n+1)\left\|T^{\prime \prime}\right\|_{p} .
$$

\section{ESTIMATES RELATED WITH CONVERGENCE FACTORS}

Proposition 4.6. The Fejér-Korovkin kernel can be written in the form

$$
K_{n}(x)=\frac{1}{2}+\sum_{k=1}^{n} \zeta_{n}\left(\frac{k}{n+2}\right) \cos (k x)=\frac{1}{2}+\sum_{k=1}^{n} \varrho_{k, n} \cos (k x) .
$$

The numbers $\varrho_{k, n}$ are usually called the convergence factors. Representations for the convergence factors of Fejér-Korovkin operators appeared in different places. For instance, see [8, p. 1098 ]. We set

$$
\zeta_{n}(x)=(1-x) \cos (\pi x)+\frac{1}{n+2} \cot \frac{\pi}{n+2} \sin (\pi x) .
$$

Corollary 4.2. For each $n>1$, one has

$$
\varrho_{1, n}=\cos \frac{\pi}{n+2}, \quad \varrho_{2, n}=1-2\left(1-\cos ^{2} \frac{\pi}{n+2}\right)\left(1-\frac{1}{n+2}\right)
$$

and

$$
4-\frac{1-\varrho_{2, n}}{1-\varrho_{1, n}}=2-2 \cos \frac{\pi}{n+2}+\frac{2(1+\cos (\pi /(n+2)))}{n+2} .
$$

We need some estimates related with the convergence factors. In what follows, we set

$$
a_{n}=\frac{1}{\pi}\left(1-\frac{\pi}{n+2} \cot \frac{\pi}{n+2}\right),
$$

$$
H_{n, 1}(x)=(1-x) \cos (\pi x)+\frac{\sin (\pi x)}{\pi}+\frac{\pi^{2}}{2} x^{2}-\frac{\pi^{2}}{3} x^{3},
$$

$$
H_{n, 2}(x)=a_{n}(\sin (\pi x)-\pi x)
$$

and

$$
\lambda_{k, n}=1-\zeta_{n}\left(\frac{k}{n+2}\right)-\frac{\pi^{2}}{2} \frac{k^{2}}{(n+2)^{2}}+\frac{\pi^{2} k^{3}}{3(n+2)^{3}}-\pi a_{n} \frac{k}{n+2} .
$$

Lemma 4.1. For $0<x \leq \pi$, one has

$$
1-\frac{x^{2}}{2} \leq x \cot x \leq 1-\frac{x^{2}}{3} .
$$

In particular,

$$
a_{n} \leq \frac{\pi}{2(n+2)^{2}} .
$$

Proof. The first assertion follows from standard arguments. For the second one,

$$
a_{n}=\frac{1}{\pi}\left(1-\frac{\pi}{n+2} \cot \frac{\pi}{n+2}\right) \leq \frac{1}{\pi} \frac{\pi^{2}}{2(n+2)^{2}} .
$$


Lemma 4.2. Let $x_{0} \in(0, \pi / 2)$ be defined by the Equation $3 \tan \left(\pi x_{0}\right)=\pi\left(1-x_{0}\right)$. For $x \in\left[0, x_{0}\right]$, one has

$$
\pi(1-x)(1-\cos (\pi x))+\sin (\pi x)-\pi x \geq 0
$$

Moreover, $x_{0}>\pi / 6$.

Proof. For $0<x \leq 1$,

$$
-4 \cos (\pi x)-\pi(1-x) \sin (\pi x) \leq 0 .
$$

Therefore, the function $f_{1}(x)=-3 \sin (\pi x)+\pi(1-x) \cos (\pi x)$ decreases in $[0,1]$. But $f_{1}\left(x_{0}\right)=0$ if and only if

$$
\tan \left(\pi x_{0}\right)=\frac{\pi}{3}\left(1-x_{0}\right)
$$

Hence, $f_{1}(x) \geq 0$ for $x \in\left[0, x_{0}\right]$.

With similar arguments, we verify that the function $f_{2}(x)=-2+2 \cos (\pi x)+\pi(1-x) \sin (\pi x)$ increases in $\left[0, x_{0}\right]$. Thus, $f_{2}(x) \geq 0$ for $x \in\left[0, x_{0}\right]$.

If $f_{3}(x)=\pi(1-x)(1-\cos (\pi x))+\sin (\pi x)-\pi x$, then $f_{3}^{\prime}(x)=\pi f_{2}(x) \geq 0$, for $x \in\left[0, x_{0}\right]$.

Lemma 4.3. Suppose that $n, m \in \mathbb{N}, n \geq m \geq 5$ and $H_{n, 1}$ is defined by (4.7), then

$$
\begin{aligned}
& \left|1-H_{n, 1}\left(\frac{m}{n+2}\right)\right| \leq \frac{\pi^{4} m^{4}}{6(n+2)^{4}}\left(\frac{1}{4}-\frac{m}{5(n+2)}\right), \\
& 0 \leq H_{n, 1}\left(\frac{m}{n+2}\right)-H_{n, 1}\left(\frac{m-1}{n+2}\right) \leq \frac{\pi^{3}(m-1)^{3}}{4(n+2)^{4}}
\end{aligned}
$$

and, for $0 \leq k \leq m-2$,

$$
\left|H_{n, 1}\left(\frac{k+2}{n+2}\right)-2 H_{n, 1}\left(\frac{k+1}{n+2}\right)+H_{n, 1}\left(\frac{k}{n+2}\right)\right| \leq \frac{\pi^{4}(k+1)^{2}}{2(n+2)^{4}} .
$$

Proof. Notice that

$$
H_{n, 1}^{\prime}(x)=-\pi(1-x) \sin (\pi x)+\pi^{2} x(1-x)=\pi(1-x)(\pi x-\sin (\pi x))
$$

and

$$
H_{n, 1}^{\prime \prime}(x)=\pi(\pi(1-x)(1-\cos (\pi x))+\sin (\pi x)-\pi x) .
$$

(i) If $x_{m}=m /(n+2)$, then

$$
\begin{aligned}
\left|1-H_{n, 1}\left(x_{m}\right)\right| & =\left|H_{n, 1}(0)-H_{n, 1}\left(x_{m}\right)\right| \\
& =\left|\int_{0}^{x_{m}} H_{n, 1}^{\prime}(s) d s\right| \\
& =\pi \int_{0}^{x_{m}}(1-s)(\pi s-\sin (\pi s)) d s .
\end{aligned}
$$

For $0 \leq y \leq 1$, one has

$$
0 \leq(1-y)(\pi y-\sin (\pi y)) \leq \frac{\pi^{3} y^{3}}{6}(1-y),
$$

hence, for $0 \leq z \leq n+2$, the function

$$
F(z)=\int_{0}^{z /(n+2)}(1-s)(\pi s-\sin (\pi s)) d s-\frac{\pi^{3}}{6(n+2)^{4}}\left(\frac{z^{4}}{4}-\frac{z^{5}}{5(n+2)}\right)
$$


decreases. Taking into account that $F(0)=0$,

$$
\left|1-H_{n, 1}\left(x_{m}\right)\right|=\pi F(m) \leq \frac{\pi^{4} m^{4}}{6(n+2)^{4}}\left(\frac{1}{4}-\frac{m}{5(n+2)}\right) .
$$

(ii) On the other hand, if $y=(m-1) /(n+2)$,

$$
\begin{aligned}
0 \leq H_{n, 1}\left(\frac{m}{n+2}\right)-H_{n, 1}\left(\frac{m-1}{n+2}\right) & =\int_{y}^{y+1 /(n+2)} H_{n, 1}^{\prime}(s) d s \\
& =\pi \int_{y}^{y+1 /(n+2)}(1-s)(\pi s-\sin (\pi s)) d s .
\end{aligned}
$$

As before, for $z \geq 0$, the function

$$
\begin{aligned}
G_{y}(z) & =\int_{y}^{y+z}(1-s)(\pi s-\sin (\pi s)) d s-\frac{\pi^{3}\left((y+z)^{4}-y^{4}\right)}{24(n+2)^{4}} \\
& =\int_{0}^{z}(1-(y+s))\left(\pi(y+s)-\sin (\pi(y+s)) d s-\frac{\pi^{3}\left((y+z)^{4}-y^{4}\right)}{24}\right.
\end{aligned}
$$

decreases and $G_{y}(0)=0$. Therefore,

$$
\begin{aligned}
H_{n, 1}\left(\frac{m}{n+2}\right)-H_{n, 1}\left(\frac{m-1}{n+2}\right) & \leq \frac{\pi^{3}\left((y+1 /(n+2))^{4}-y^{4}\right)}{24} \\
& =\frac{\pi^{3}}{24}\left(4 \frac{y^{3}}{(n+2)}+6 \frac{y^{2}}{(n+2)^{2}}+4 \frac{y}{(n+2)^{3}}+\frac{1}{(n+2)^{4}}\right) \\
& =\frac{\pi^{3}}{24(n+2)^{4}}\left(4(m-1)^{3}+6(m-1)^{2}+4(m-1)+1\right) \\
& =\frac{\pi^{3}(m-1)^{3}}{24(n+2)^{4}}\left(4+\frac{6}{(m-1)}+\frac{4}{(m-1)^{2}}+\frac{1}{(m-1)^{3}}\right) \\
& \leq \frac{\pi^{3}(m-1)^{3}}{4(n+2)^{4}}
\end{aligned}
$$

for $m \geq 5$.

(iii) Let $x_{0}$ be given as in Lemma 4.2. Set $z=k /(n+2)$. Note that

$$
0 \leq z<z+\frac{2}{n+2} \leq \frac{m-2+2}{n+2} \leq \frac{\pi}{6}<x_{0} .
$$

Hence, if $0 \leq s \leq z+2 /(n+2)$,

$$
\begin{aligned}
0 & \leq \frac{1}{\pi} H_{n, 1}^{\prime \prime}(s) \\
& =\pi(1-s)(1-\cos (\pi s))+\sin (\pi s)-\pi s \\
& \leq \pi(1-\cos (\pi s)) \\
& <\pi \frac{(\pi s)^{2}}{2} .
\end{aligned}
$$


Therefore,

$$
\begin{aligned}
0 & \leq H_{n, 1}(z)-2 H_{n, 1}\left(z+\frac{1}{n+2}\right)+H_{n, 1}\left(z+\frac{2}{n+2}\right) \\
& =\int_{0}^{1 /(n+2)}\left(H_{n, 1}^{\prime}(z+s+1 /(n+2))-H_{n, 1}^{\prime}(z+s)\right) d s \\
& =\int_{0}^{1 /(n+2)} \int_{0}^{1 /(n+2)} H_{n, 1}^{\prime \prime}(z+s+t) d t d s \\
& \leq \frac{\pi^{4}}{2} \int_{0}^{1 /(n+2)} \int_{0}^{1 /(n+2)}(z+s+t)^{2} d t d s \\
& =\frac{\pi^{4}}{6} \int_{0}^{1 /(n+2)}\left(3 \frac{(z+s)^{2}}{n+2}+3 \frac{(z+s)}{(n+2)^{2}}+\frac{1}{(n+2)^{3}}\right) d s \\
& =\frac{\pi^{4}}{6}\left(\frac{1}{n+2}\left(\frac{3 z^{2}}{n+2}+\frac{3 z}{(n+2)^{2}}+\frac{1}{(n+2)^{3}}\right)+\frac{3}{2(n+2)^{3}}\left(2 z+\frac{1}{n+2}\right)+\frac{1}{(n+2)^{4}}\right) \\
& =\frac{\pi^{4}}{6(n+2)^{4}}\left(3 k^{2}+3 k+1+3 k+\frac{3}{2}+1\right) \\
& =\frac{\pi^{4}}{6(n+2)^{4}}\left(3 k^{2}+6 k+\frac{5}{2}\right) \\
& <\frac{\pi^{4}}{6(n+2)^{4}}\left(3 k^{2}+6 k+3\right) \\
& =\frac{\pi^{4}}{2(n+2)^{4}}(k+1)^{2} .
\end{aligned}
$$

Lemma 4.4. If $n, m \in \mathbb{N}, n \geq m \geq 5$, and $H_{n, 2}$ is defined by (4.7), then

$$
\begin{gathered}
\left|H_{n, 2}\left(\frac{m}{n+2}\right)\right| \leq \frac{\pi^{4}}{12} \frac{m^{3}}{(n+2)^{5}} \\
\left|H_{n, 2}\left(\frac{m-1}{n+2}\right)-H_{n, 2}\left(\frac{m}{n+2}\right)\right| \leq \frac{\pi^{4}}{4} \frac{m^{2}}{(n+2)^{5}},
\end{gathered}
$$

and, for $0 \leq k \leq m-2$,

$$
\left|H_{n, 2}\left(\frac{k+2}{n+2}\right)-2 H_{n, 2}\left(\frac{k-1}{n+2}\right)+H_{n, 2}\left(\frac{k}{n+2}\right)\right| \leq \frac{\pi^{4}\left(3 k^{2}+9 k+7\right)}{4(n+2)^{5}} .
$$

Proof. (i) If $x=m /(n+2)$, one has

$$
\begin{aligned}
\left|H_{n, 2}(x)\right| & =\frac{1}{\pi}\left(1-\frac{\pi}{n+2} \cot \frac{\pi}{n+2}\right)(\pi x-\sin (\pi x)) \\
& \leq \frac{1}{\pi} \frac{\pi^{2}}{2(n+2)^{2}} \frac{(\pi x)^{3}}{6} \\
& =\frac{\pi^{4}}{12} \frac{m^{3}}{(n+2)^{5}}
\end{aligned}
$$


(ii) On the other hand, there exists $\theta \in(\pi(m-1) /(n+2), \pi m /(n+2))$ such that (see Lemma 4.1)

$$
\begin{aligned}
\left|H_{n, 2}\left(\frac{m-1}{n+2}\right)-H_{n, 2}\left(\frac{m}{n+2}\right)\right| & =a_{n}\left|\sin \left(\frac{\pi m}{n+2}\right)-\sin \left(\frac{\pi(m-1)}{n+2}\right)-\frac{\pi}{n+2}\right| \\
& =\frac{\pi a_{n}}{n+2}(1-\cos \theta) \\
& \leq \frac{\pi a_{n}}{n+2} \frac{\theta^{2}}{2} \\
& \leq \frac{\pi^{4}}{4(n+2)^{3}}\left(\frac{m}{n+2}\right)^{2} \\
& \leq \frac{\pi^{4}}{4} \frac{m^{2}}{(n+2)^{5}} .
\end{aligned}
$$

Finally, taking into account Lemma 4.1 and setting $z=k /(n+2)$, one has

$$
\begin{aligned}
& \left|H_{n, 2}(z+2 /(n+2))-2 H_{n, 2}(z+1 /(n+2))+H_{n, 2}(z)\right| \\
= & a_{n} \mid \sin (\pi(z+2 /(n+2))-2 \sin (\pi(z+1 /(n+2))+\sin (\pi z)) \mid \\
= & \pi^{2} a_{n}\left|\int_{0}^{1 /(n+2)} \int_{0}^{1 /(n+2)} \sin (\pi(z+s+t)) d s d t\right| \\
= & \pi a_{n} \int_{0}^{1 /(n+2)}\left(1-\cos \left(\pi\left(z+s+\frac{1}{n+2}\right)\right)\right) d s \\
\leq & \frac{\pi^{4}}{4(n+2)^{2}} \int_{0}^{1 /(n+2)}\left(z+s+\frac{1}{n+2}\right)^{2} d s \\
= & \frac{\pi^{4}}{4(n+2)^{2}}\left(\frac{3}{n+2}\left(z+\frac{1}{n+2}\right)^{2}+\frac{3}{(n+2)^{2}}\left(z+\frac{1}{n+2}\right)+\frac{1}{(n+2)^{3}}\right) \\
= & \frac{\pi^{4}}{4(n+2)^{5}}\left(3(k+1)^{2}+3(k+1)+1\right) \\
= & \frac{\pi^{4}}{4(n+2)^{5}}\left(3 k^{2}+9 k+7\right) .
\end{aligned}
$$

Lemma 4.5. If $n, m \in \mathbb{N}, 5 \leq m \leq n$ and $\lambda_{k, n}$ is defined by (4.9), then

$$
\left|\lambda_{m, n}\right| \leq \frac{\pi^{4}}{24} \frac{m^{4}}{(n+2)^{4}}, \quad\left|m\left(\lambda_{m-1, n}-\lambda_{m, n}\right)\right| \leq \frac{\pi^{3} m^{4}}{4(n+2)^{4}}
$$

and

$$
\left|\sum_{k=0}^{m-2}(k+1) \Delta^{2} \lambda_{k, n+2}\right|<\frac{\pi^{4} m^{3}(m-1)}{(n+2)^{4}}
$$

Proof. Notice that

$$
\begin{aligned}
1-H_{n, 1}(x)+H_{n, 2}(x) & =1-(1-x) \cos (\pi x)-\frac{\sin (\pi x)}{\pi}-\frac{\pi^{2}}{2} x^{2}+\frac{\pi^{2}}{3} x^{3}+a_{n}(\sin (\pi x)-\pi x) \\
& =1-(1-x) \cos (\pi x)-\frac{\pi}{n} \cot \frac{\pi}{n} \sin (\pi x)-\frac{\pi^{2}}{2} x^{2}+\frac{\pi^{2}}{3} x^{3}-a_{n} \pi x .
\end{aligned}
$$


Therefore,

$$
\lambda_{k, n}=1-H_{n, 1}\left(\frac{k}{n+2}\right)+H_{n, 2}\left(\frac{k}{n+2}\right) .
$$

(i) If $m \geq 5$, it follows from Lemmas 4.3 and 4.4 that

$$
\begin{aligned}
\left|\lambda_{m, n}\right| & =\left|1-H_{n, 1}\left(\frac{m}{n+2}\right)+H_{n, 2}\left(\frac{m}{n+2}\right)\right| \\
& \leq \frac{\pi^{4}}{6} \frac{m^{4}}{(n+2)^{4}}\left(\frac{1}{4}-\frac{m}{5(n+2)}\right)+\frac{\pi^{4}}{12} \frac{m^{3}}{(n+2)^{5}} \\
& \leq \frac{\pi^{4}}{24} \frac{m^{4}}{(n+2)^{4}} .
\end{aligned}
$$

(ii) On the other hand,

$$
\begin{aligned}
\left|m\left(\lambda_{m-1, n}-\lambda_{m, n}\right)\right| & \leq m\left|H_{n, 1}\left(\frac{m}{n+2}\right)-H_{n, 1}\left(\frac{m-1}{n+2}\right)\right|+m\left|H_{n, 2}\left(\frac{m}{n+2}\right)-H_{n, 2}\left(\frac{m-1}{n+2}\right)\right| \\
& \leq \frac{\pi^{3} m(m-1)^{3}}{4(n+2)^{4}}+\frac{\pi^{4}}{4} \frac{m^{3}}{(n+2)^{5}} \\
& \leq \frac{\pi^{3} m^{3}}{4(n+2)^{4}}\left(m-1+\frac{\pi}{(n+2)}\right) \\
& \leq \frac{\pi^{3} m^{4}}{4(n+2)^{4}}
\end{aligned}
$$

(iii) Finally, for $0 \leq k \leq m-2$, then

$$
\begin{aligned}
\left|\Delta^{2} \lambda_{k, n+2}\right| & \leq\left|H_{n, 1}\left(\frac{k+2}{n+2}\right)-2 H_{n, 1}\left(\frac{k+1}{n+2}\right)+H_{n, 1}\left(\frac{k}{n+2}\right)\right| \\
& +\left|H_{n, 2}\left(\frac{k+2}{n}\right)-2 H_{n, 2}\left(\frac{k+1}{n}\right)+H_{n, 2}\left(\frac{k}{n}\right)\right| \\
& \leq \frac{\pi^{4}(k+1)^{2}}{2(n+2)^{4}}+\frac{\pi^{4}\left(3 k^{2}+9 k+7\right)}{4(n+2)^{5}} \\
& \leq \frac{\pi^{4}}{(n+2)^{4}}\left(4 k^{2}+11 k+8\right) .
\end{aligned}
$$

Since

$$
\begin{aligned}
\sum_{k=0}^{m-2}(k+1)\left(4 k^{2}+11 k+8\right) & =\sum_{k=0}^{m-2}\left(4 k^{3}+15 k^{2}+19 k+8\right) \\
& =(m-1)(m-2)\left((m-2)(m-1)+15 \frac{(2 m-3)}{6}+\frac{19}{2}+\frac{8}{m-2}\right) \\
& =m^{4}-m^{3} .
\end{aligned}
$$

Therefore,

$$
\left|\sum_{k=0}^{m-2}(k+1) \Delta^{2} \lambda_{k, n+2}\right| \leq \frac{\pi^{4} m^{3}(m-1)}{(n+2)^{4}}
$$




\section{MAin RESUlts}

Theorem 5.6. Assume $1 \leq p \leq \infty, m \in \mathbb{N}$ and $m \geq 2$. If $g \in W_{p}^{2}, T \in \mathbb{T}_{m}$, and $\|g-T\|_{p}=E_{m, p}(g)$, then

$$
\left\|g^{\prime \prime}-T^{\prime \prime}\right\|_{p} \leq 30 E_{m, p}\left(g^{\prime \prime}\right)
$$

Proof. It was proved in [1, Th. 3.4] that, for any $r \in \mathbb{N}, g \in W_{p}^{r}, T \in \mathbb{T}_{m}$, and $m \geq \max \{2, r\}$.

$$
\left\|D^{r}(g)-D^{r}(S)\right\|_{p} \leq\left(4+\frac{1}{r}+\ln (2 r)\right)\left(1+\frac{e \pi}{2}\right) E_{m, p}\left(D^{r}(g)\right)
$$

when $\|g-S\|_{p}=E_{m, p}(g)$. Since

$$
\left(4+\frac{1}{2}+\ln (4)\right)\left(1+\frac{e \pi}{2}\right)<30
$$

one has

$$
\left\|D^{2}(g)-D^{2}(T)\right\|_{p} \leq 30 E_{m, p}\left(D^{2}(g)\right)
$$

Proposition 5.7. If $1 \leq p \leq \infty g \in W_{p}^{2}$, then

$$
\left\|g-\mathbb{F}_{n}(g)\right\|_{p} \leq \frac{\pi^{4}}{4(n+2)^{2}}\left\|D^{2}(g)\right\|_{p}
$$

Proof. It follows from Proposition 2.2 and Corollary 4.2 that

$$
\begin{aligned}
\left\|g-\mathbb{F}_{n}(g)\right\|_{p} & \leq \frac{\pi^{2}}{2}\left(1-\varrho_{1, n}\right)\left\|D^{2}(g)\right\|_{p} \\
& =\frac{\pi^{2}}{2}\left(1-\cos \frac{\pi}{n+2}\right)\left\|D^{2}(g)\right\|_{p} \\
& \leq \frac{\pi^{4}}{4(n+2)^{2}}\left\|D^{2}(g)\right\|_{p} .
\end{aligned}
$$

Proposition 5.8. For each $1 \leq p \leq \infty$, if $n, m \in \mathbb{N}, n \geq m \geq 5$ and $T \in \mathbb{T}_{m}^{0}$, then

$$
\left\|\mathbb{F}_{n}(T)-T-\frac{\pi^{2}}{2(n+2)^{2}} T^{\prime \prime}\right\|_{p} \leq\left(\frac{9 \pi^{3} m^{4}}{(n+2)^{4}}+\frac{3 \pi^{2}}{4(n+2)^{3}}+\frac{2 \pi^{2}(m+1)}{3(n+2)^{3}}\right)\left\|T^{\prime \prime}\right\|_{p} .
$$


Proof. Let $\lambda_{k, n}$ be defined by (4.9). If $T=\sum_{k=0}^{m} A_{k}(x) \in \mathbb{T}_{n}$, with $A_{k}(x)=a_{k} \cos (k x)+$ $b_{k} \sin (k x)$, then

$$
\begin{aligned}
& T(x)-\mathbb{F}_{n}(T, x)+\frac{\pi^{2}}{2(n+2)^{2}} T^{\prime \prime}(x) \\
= & \sum_{k=0}^{m}\left(1-\zeta_{n+2}\left(\frac{k}{n+2}\right)-\frac{\pi^{2} k^{2}}{2(n+2)^{2}}\right) A_{k}(x) \\
= & \sum_{k=0}^{m}\left(1-\zeta_{n+2}\left(\frac{k}{n+2}\right)-\frac{\pi^{2}}{2} \frac{k^{2}}{(n+2)^{2}}+\frac{\pi^{2} k^{3}}{3(n+2)^{3}}-\pi a_{n} \frac{k}{n+2}\right) A_{k}(x) \\
+ & \sum_{k=1}^{m}\left(\pi a_{n} \frac{k}{n+2}-\frac{\pi^{2} k^{3}}{3(n+2)^{3}}\right) A_{k}(x) \\
= & \sum_{k=0}^{m} \lambda_{k, n} A_{k}(x)+\frac{\pi a_{n}}{n+2} \widetilde{T}^{\prime}-\frac{\pi^{2}}{3(n+2)^{3}} \widetilde{T}^{\prime \prime \prime} .
\end{aligned}
$$

We apply twice the Abel transformation to obtain

$$
\begin{aligned}
\sum_{k=0}^{m} \lambda_{k, n} A_{k}(x) & =\lambda_{m, n} \sum_{k=0}^{m} A_{k}(x)+\sum_{k=0}^{m-1}\left(\lambda_{k, n}-\lambda_{k+1, n}\right) \sum_{j=0}^{k} A_{j}(x) \\
& =\lambda_{m, n} T(x)+\left(\lambda_{m-1, n}-\lambda_{m, n}\right) \sum_{k=0}^{m-1} \sum_{j=0}^{k} A_{k}(x)+\sum_{k=0}^{m-2} \Delta^{2} \lambda_{k, n} \sum_{j=0}^{k} \sum_{i=0}^{j} A_{i}(x) \\
& =\lambda_{m, n} T(x)+m\left(\Delta^{1} \lambda_{m-1, n}\right) \sigma_{m}(T, x)+\sum_{k=0}^{m-2}(k+1) \Delta^{2} \lambda_{k, n} \sigma_{k}(T, x),
\end{aligned}
$$

where $\sigma_{k}$ is the Fejér sum. It follows from Lemma 4.5 that

$$
\begin{aligned}
\left\|\sum_{k=0}^{n} \lambda_{k, n} A_{k}(x)\right\|_{p} & \leq\left(\frac{\pi^{4}}{24} \frac{m^{4}}{(n+2)^{4}}+\frac{\pi^{3} m^{4}}{4(n+2)^{4}}+\frac{\pi^{4} m^{3}(m-1)}{(n+2)^{4}}\right)\|T\|_{p} \\
& \leq\left(\frac{1}{24} \frac{16}{5}+\frac{1}{4}+\frac{16}{5}\right) \frac{\pi^{4} m^{4}}{(n+2)^{4}}\|T\|_{p} \\
& \leq \frac{\pi^{3} m^{4}}{(n+2)^{4}}\|T\|_{p} \\
& \leq \frac{4 \pi^{3} m^{4}}{(n+2)^{4}}\|T\|_{p} .
\end{aligned}
$$

Now, taking into account Lemma 4.1 , we obtain

$$
\begin{aligned}
\left\|T-\mathbb{F}_{n}(T)+\frac{\pi^{2}}{2(n+2)^{2}} T^{\prime \prime}\right\|_{p} & \leq\left\|\sum_{k=0}^{m} \lambda_{k, n} A_{k}(x)\right\|+\frac{\pi a_{n}\left\|\widetilde{T}^{\prime}\right\|_{p}}{n+2}-\frac{\pi^{2}\left\|\widetilde{T}^{\prime \prime \prime}\right\|_{p}}{3(n+2)^{3}} \\
& \leq \frac{4 \pi^{3} m^{4}}{(n+2)^{4}}\|T\|_{p}+\frac{\pi^{2}}{2(n+2)^{3}}\left\|\widetilde{T}^{\prime}\right\|_{p}+\frac{\pi^{2}}{3(n+2)^{3}}\left\|\widetilde{T}^{\prime \prime \prime}\right\|_{p} .
\end{aligned}
$$

Each one of the norms given above can be estimated with the help of Corollary 3.1 (here the condition $T \in \mathbb{T}_{m}^{0}$ is needed). That is

$$
\left\|T-\mathbb{F}_{n}(T)+\frac{\pi^{2}}{2(n+2)^{2}} T^{\prime \prime}\right\|_{p} \leq\left(\frac{9 \pi^{3} m^{4}}{(n+2)^{4}}+\frac{3 \pi^{2}}{4(n+2)^{3}}+\frac{2 \pi^{2}(m+1)}{3(n+2)^{3}}\right)\left\|T^{\prime \prime}\right\|_{p} .
$$


Theorem 5.7. Assume $1 \leq p \leq \infty$ and $0<\alpha<1$. If $n+2 \geq 7^{2 / \alpha}$ and $f \in W_{p}^{2}$, then

$$
\left\|(n+2)^{2}\left(\mathbb{F}_{n-2}(f)-f\right)-\frac{\pi^{2}}{2} f^{\prime \prime}\right\|_{p} \leq \frac{795 \pi^{2}}{(n+2)^{2 / 3}}\left\|f^{\prime \prime}\right\|_{p} .
$$

Proof. Fix $m \in \mathbb{N}$ such that

$$
m+1=\left[(n+2)^{\alpha / 2}\right] .
$$

Note that

$$
6 \leq(n+2)^{\alpha / 2}-1<\left[(n+2)^{\alpha / 2}\right]=m+1<n .
$$

Let $T_{m} \in \mathbb{T}_{m}$ be given by the condition $E_{m . p}(f)=\left\|f-T_{m}\right\|_{p}$. Since

$$
(n+2)^{2}\left(\mathbb{F}_{n}(f+c)-(f+c)\right)-\frac{\pi^{2}}{2}(f+c)^{\prime \prime}=(n+2)^{2}\left(\mathbb{F}_{n}(f)-f\right)-\frac{\pi^{2}}{2} f^{\prime \prime}
$$

and

$$
E_{m, p}(f+c)=E_{m, p}(f),
$$

for any real constant $c$, without losing generality, we can assume that $T_{m}$ has mean zero. That is $T_{m} \in \mathbb{T}_{m}^{0}$. Taking into account Proposition 5.7 and (5.10),

$$
\begin{aligned}
\left\|(n+2)^{2}\left(\mathbb{F}_{n}(f)-f\right)-\frac{\pi^{2}}{2} f^{\prime \prime}\right\|_{p} & \leq(n+2)^{2}\left\|\mathbb{F}_{n}\left(f-T_{m}\right)-\left(f-T_{m}\right)\right\|_{p} \\
& +\frac{\pi^{2}}{2}\left\|f^{\prime \prime}-T_{m}^{\prime \prime}\right\|_{p}+\left\|(n+2)^{2}\left(\mathbb{F}_{n}\left(T_{m}\right)-T_{m}\right)-\frac{\pi^{2}}{2} T_{m}^{\prime \prime}\right\|_{p} \\
& \leq\left(\frac{\pi^{4}}{4}+\frac{\pi^{2}}{2}\right)\left\|f^{\prime \prime}-T_{m}^{\prime \prime}\right\|_{p}+\left\|(n+2)^{2}\left(\mathbb{F}_{n}\left(T_{m}\right)-T_{m}\right)-\frac{\pi^{2}}{2} T_{m}^{\prime \prime}\right\|_{p} \\
& \leq 30\left(\frac{\pi^{4}}{4}+\frac{\pi^{2}}{2}\right) E_{m, p}\left(f^{\prime \prime}\right)+\left\|(n+2)^{2}\left(\mathbb{F}_{n}\left(T_{m}\right)-T_{m}\right)-\frac{\pi^{2}}{2} T_{m}^{\prime \prime}\right\|_{p} .
\end{aligned}
$$

From Proposition 5.8, we know that

$$
\begin{aligned}
& \left\|(n+2)^{2}\left(\mathbb{F}_{n}\left(T_{m}\right)-T_{m}\right)-\frac{\pi^{2}}{2} T_{m}^{\prime \prime}\right\|_{p} \\
\leq & \left(\frac{9 \pi^{3} m^{4}}{(n+2)^{2}}+\frac{3 \pi^{2}}{4(n+2)}+\frac{2 \pi^{2}(m+1)}{3(n+2)}\right)\left\|T_{m}^{\prime \prime}\right\|_{p} \\
\leq & \left(\frac{9 \pi^{3}(n+2)^{2 \alpha}}{(n+2)^{2}}+\frac{3 \pi^{2}}{4(n+2)}+\frac{2 \pi^{2}(n+2)^{\alpha / 2}}{3(n+2)}\right)\left(\left\|T_{m}^{\prime \prime}-f^{\prime \prime}\right\|_{p}+\left\|f^{\prime \prime}\right\|_{p}\right) \\
\leq & \left(\frac{9 \pi^{3}}{(n+2)^{2(1-\alpha)}}+\frac{3 \pi^{2}}{4(n+2)}+\frac{2 \pi^{2}}{3(n+2)^{1-\alpha / 2}}\right)\left(30 E_{m, p}\left(f^{\prime \prime}\right)+\left\|f^{\prime \prime}\right\|_{p}\right) \\
\leq & 31 \pi^{2}\left(9 \pi+\frac{3}{4}+\frac{2}{3}\right) \frac{\left\|f^{\prime \prime}\right\|_{p}}{(n+2)^{\alpha}} \\
\leq & (31 \pi)^{2} \frac{\left\|f^{\prime \prime}\right\|_{p}}{(n+2)^{\alpha}} .
\end{aligned}
$$


Moreover, taking into account Theorem 2.4, one has

$$
\begin{aligned}
30\left(\frac{\pi^{4}}{4}+\frac{\pi^{2}}{2}\right) E_{m, p}\left(f^{\prime \prime}\right) & \leq 150 \pi^{2}\left(\frac{10}{4}+\frac{1}{2}\right) \omega_{1}\left(f^{\prime \prime}, \frac{2 \pi}{m+1}\right)_{p} \\
& =450 \pi^{2} \omega_{1}\left(f^{\prime \prime}, \frac{2 \pi}{m+1}\right)_{p} \\
& \leq 450 \pi^{2} \omega_{1}\left(f^{\prime \prime}, \frac{2 \pi}{(n+2)^{\alpha / 2}-1}\right)_{p} .
\end{aligned}
$$

We have proved that

$$
\left\|(n+2)^{2}\left(\mathbb{F}_{n}(f)-f\right)-\frac{\pi^{2}}{2} f^{\prime \prime}\right\|_{p} \leq 450 \pi^{2} \omega_{1}\left(f, \frac{2 \pi}{(n+2)^{\alpha / 2}-1}\right)_{p}+\frac{(31 \pi)^{2}}{(n+2)^{\alpha}}\left\|f^{\prime \prime}\right\|_{p} .
$$

This yields the result.

\section{REFERENCES}

[1] J. Bustamante, L. Flores-de-Jesús: Strong converse inequalities and quantitative Voronovskaya-type theorems for trigonometric Fejér sums. Constr. Math. Anal. 3 (2) (2020), 53-63.

[2] P. L. Butzer, E. Gorlich: Saturationsklassen und asymptotische Eigenschaften trigonometrischer singulärer Integrale. (German), 1966 Festschr. Gedächtnisfeier K. Weierstrass, Westdeutscher Verlag, Cologne, 339-392.

[3] P. L. Butzer, R. J. Nessel: Fourier Analysis and Approximation. New York-Base1 (1971).

[4] P. L. Butzer, E. L. Stark: On a trigonometric convolution operator with kernels having two zeros of simple multiplicity. Acta Math. Acad. Sci. Hung. 20 (1969), 451-461.

[5] S. Foucart, Y. Kryakin and A. Shadrin: On the exact constant in the Jackson-Stechkin inequality for the uniform metric. Constr. Approx. 29 (2009), 157-179.

[6] P. P. Korovkin: An asymptotic property of positive methods of summation of Fourier series and best approximation of functions of class $Z_{2}$ by linear positive polynomial operators. (in Russian), Uspehi Mat. Nauk 6 (84) (1958), 99-103.

[7] I. M. Petrov: Order of approximation of functions of the class Z for some polynomial operators. (in Russian), Uspehi Mat. Nauk 13 (84) (1958), 127-131.

[8] E. L. Stark: The kernel of Fejér-Korovkin: a basic tool in the constructive theory of functions. Functions, series, operators, Vol. I, II (Budapest, 1980), Colloq. Math. Soc. János Bolyai, 35, North-Holland, Amsterdam, 1983, 1095-1123.

[9] A. F. Timan: Theory of Approximation of Functions of Real Variable. Pergamon Press, 1963.

[10] A. Zygmund: Trigonometric series. Third Edition, Vol I and II combined, Cambridge Mathematical Library, 2002.

JORGE BUSTAMANTE

BENEMÉRITA Universidad AutónOMa de Puebla

Facultad de Ciencias Físico-Matemáticas

C.P. 72570 , PuEbla, MÉxico.

ORCID: 0000-0003-2856-6738

E-mail address: jbusta@f $\mathrm{ffm} . \mathrm{buap} . \mathrm{mx}$

LÁZARO FLORES-DE-JESÚS

BENEMÉRITA Universidad AutónOMa de Puebla

Facultad de Ciencias Físico-Matemáticas

C.P. 72570 , PUEbla, MÉXICO.

ORCID: 0000-0002-3431-5903

E-mail address: lazarofdj@gmail.com 